\title{
Rollin' Justin - Design considerations and realization of a mobile platform for a humanoid upper body
}

\author{
M. Fuchs, Ch. Borst, P. Robuffo Giordano, A. Baumann, E. Kraemer, J. Langwald, R. Gruber, \\ N. Seitz, G. Plank, K. Kunze, R. Burger, F. Schmidt, T. Wimboeck and G. Hirzinger \\ German Aerospace Center - Institute of Robotics and Mechatronics \\ Oberpfaffenhofen, Germany, 2008 \\ Email: matthias.fuchs@dlr.de
}

\begin{abstract}
Research on humanoid robots for use in servicing tasks, e.g. fetching and delivery, attracts steadily more interest. With Rollin' Justin a mobile robotic system and research platform is presented that allows the implementation and demonstration of sophisticated control algorithms and dexterous manipulation. Important problems of service robotics such as mobile manipulation and strategies for using the increased workspace and redundancy in manipulation task can be studied in detail. This paper gives an overview of the design considerations for a mobile platform and their realizations to transform the formerly table-mounted humanoid upper body system Justin into Rollin' Justin, a fully self-sustaining mobile research platform.
\end{abstract}

\section{INTRODUCTION}

In 2006 the humanoid upper body Justin was presented as a research platform for dual arm manipulation. Using its two arms and hands, controlled as hardware in the loop system, some basic control schemes for robust dual handed dual armed manipulation tasks have been evaluated and published. To further enhance the system's field of work it was soon considered to equip it with a mobile base. This would allow using Justin also as a platform for mobile manipulation to study strategies utilizing the increased workspace and redundancy for manipulation tasks. Also a mobile Justin serves perfect as a service robotic prototype to demonstrate enhanced autonomy features. This paper shows the design considerations we took into account to build Justin's mobile platform as well as some of the technical details of the realized robotic system.

\section{BASIC DESIGN CONSIDERATIONS}

Obviously the most adequate enhancement for "Justin" would be legs to make him walk and therefore a full humanoid platform. However human inspired legs will drastically increase the systems complexity adding at least 10 to 12 degrees of freedom (DOF) to the already existing 43 DOF of the upper body. Also it would probably turn the research attention too much to walking and dynamical stability issues than to the enhancement of the dual handed manipulation capabilities. Therefore only wheeled solutions for the mobile base where considered.

The basic design considerations ordered by their importance can be summarized as follows:

(1) Fulfill functional needs of the upper body

(2) Prefer compact construction

(3) Usage of off-the-shelf components

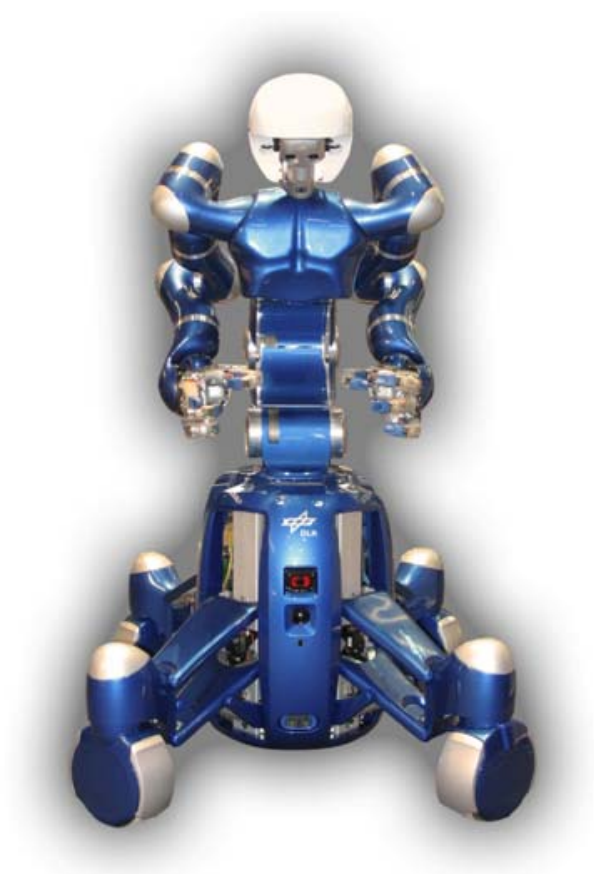

Fig. 1: The Rollin’ Justin

The mobile base is intended as an enhancement of the system and should not restrain the capabilities of the upper body. The large workspace and workload as well as the high dynamic properties of Justin for example should not be limited by the platform. This imposes high demands on the static or dynamic stability of the base. On the other hand the platform should be designed as compact as possible to pass through standard doorways and to make the appearance of the whole system pleasant and nonhazardous. To decrease system complexity, cost and project duration standard components are to be used. The most preferred way would be to integrate the system on an available mobile platform that can fulfill the functional needs.

\section{A. Functional needs and available platforms}

The upper body system itself has the mass, payload and power consumption properties as listed in table 1 . The platform must be able to preserve active or passive (static) stability to the system if Justin lifts objects up to $20 \mathrm{~kg}$ from the floor. The power supply must provide up to $1 \mathrm{~kW}$ for the actuation system and at least two computers for control and perception of the upper body. For the convenient usage of the base as workspace extension during manipulation tasks, e.g. in front of a desk, the mobile drive system should be at 
least omnidirectional. To reach the best performance in the control of the total system the mobile base should allow closed loop control together with torso and arms within a bandwidth of $100 \mathrm{~Hz}$ minimum in hard realtime.

TABLE 1

\begin{tabular}{|l|l|l|l|}
\hline Component & \multicolumn{1}{l}{ Mass } & \multicolumn{1}{l}{ Payload } & \multicolumn{1}{l}{ Power Cons. } \\
\hline Hand & $1.8 \mathrm{~kg}$ & $3 \mathrm{~kg}$ (Tip) & $15-50 \mathrm{~W}$ \\
\hline Arm & $13 \mathrm{~kg}$ & $15 \mathrm{~kg}$ (static) & $50-150 \mathrm{~W}$ \\
\hline Torso+Head & $15 \mathrm{~kg}$ & $50 \mathrm{~kg}$ (static) & $100-250 \mathrm{~W}$ \\
\hline Total & $\sim 50 \mathrm{~kg}$ & $\sim 20 \mathrm{~kg}$ (static) & $0.5-1 \mathrm{~kW}$ \\
\hline
\end{tabular}

An intensive review of existing and commercial available platforms [1] showed that most mobile robots are based on a statically stable platform principle. This means that no control or force/torque input is needed for maintaining stability. Within this group of platforms different wheels or tracks for propulsion are used. The number of legs equipped with wheels varies from two with one passive wheel (eg. Pioneer P3-DX, MetraLabs SCITOS G5), to be statically stable, over four (Pioneer P3-AT) and six [2], usually used for off terrain vehicles, up to eight [3] wheels. It should be noted that vehicles with more than three wheels are hyper static and require some form of suspension if all wheels are to maintain good ground contact. Not many of the previously designed smaller platforms can carry higher loads as required for Justin except of some platforms dedicated directly to carry upper bodies or industrial robot arms like the Hermes [4], the ARMAR platform [5], the Twendy-One [6] or the Nomadic XR4000 [7]. Actually none of them are commercial available.

Recently the dynamically stabilized two wheeled platform principle well known from the Segway RMP has been used for the integration of some manipulators like the NASA Robonaut [8] or the Toyota Work Partner Robot. An advantage of such a system is the small weight and footprint of the platform but the power consumption and complexity is higher, safety issues in case of control failures are to be tracked and for manipulation actions the most critical factor is that the platform reacts negatively on stiff contacts with fixed objects in the environment.

It can be stated that no off-the-shelf concept for a suitable mobile platform for Justin existed. In consequence a new platform has been developed.

\section{B. The Platform Concept for Justin}

To keep the design of the platform as simple as possible a static base with four wheels was considered. To meet the stability requirements during the manipulation tasks with high load and high dynamics as well as the need for compact design to move through narrow passages a variable footprint mechanism was envisaged [1]. To allow easy integration of the platform kinematics the base should support omnidirectional movements. Further the power supply of the mobile base must also serve the upper body. The necessary computation platforms and communication equipment have to be integrated as well. The result of the development of a mobile base for Justin is shown in Fig. 1.

In the following we introduce Justin's mobile platform and its technical details. Section III describes the mechanical structure and its main components. The electronics power supply devices are shown in Sec. IV followed by the communication concept (V) and some considerations on the platform control (VI). A conclusion is given in Sec. 0 .

\section{MECHANICAL DESIGN}

The two-arm system Justin is a powerful upper body humanoid robot that is able to lift weights up to $20 \mathrm{~kg}$. Its arm span is $3000 \mathrm{~mm}$ and the torso can move about $600 \mathrm{~mm}$ to the front and $300 \mathrm{~mm}$ to the back [9]. The whole upper body can rotate 170 degree to both sides relative to its base. According to its weight (about $50 \mathrm{~kg}$ ), workspace and force this robot has to be mounted on a very stable base with an adequate footprint to prevent the robot from falling over.

The necessary footprint (maximum supporting polygon) was evaluated by analyzing different torso positions (arms vertical or horizontal oriented) carrying a payload of $3 \mathrm{~kg}$ and at different accelerations [1]. The torques applied on the last axis of the torso ranged from $248 \mathrm{Nm}$ in the static case up to $1800 \mathrm{Nm}$. In any of these cases the mass and support polygon of the base should prevent tumbling down the system.

The minimal footprint can be evaluated in a similar way assuming a compact configuration of Justin in the static case. Practically the minimal size of the platform is determined by the volume needed for the power supply, electronics and computer components to make it a fully autonomous system.

The wheel extension mechanism is designed to be a very compact construction with highly integrated components. Due to the actuation by the wheels of the platform themselves, no extra motors are necessary for the extension and retraction function.
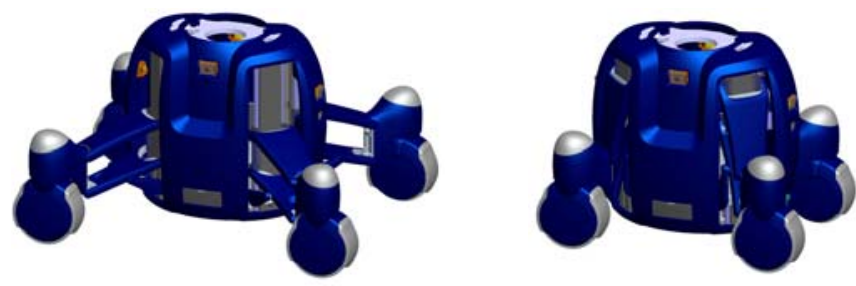

Fig. 2: Platform with extended and retracted wheels

Fig. 2 shows the DLR mobile platform with extended and retracted wheels. It has a maximum length of $1220 \mathrm{~mm}$ and a maximum width of $1052 \mathrm{~mm}$. The minimum dimensions are $812 \mathrm{~mm} \times 644 \mathrm{~mm}$. As the platform is equipped with spring damped wheel suspensions its height varies between $658 \mathrm{~mm}$ and $728 \mathrm{~mm}$. The weight is about $150 \mathrm{~kg}$.

With extended wheels the platform has a footprint of $985 \mathrm{~mm}$ x $815 \mathrm{~mm}$. The decisive factor for the maximum footprint is the stability. Together with a very low center of gravity (about $260 \mathrm{~mm}$ above the ground quite centered to the footprint) this footprint is enough to avoid a tilt over even in case of an emergency stop at full speed with the upper body bent forward [1]. By retraction of the wheels towards the platform the footprint can be reduced to $685 \mathrm{~mm}$ x $515 \mathrm{~mm}$ (cf. Fig. 3). Here the decisive factor is the possibility to pass doors. While the wheels are retracted, the 
platform is able to go to every place where a wheel chair can go as well.

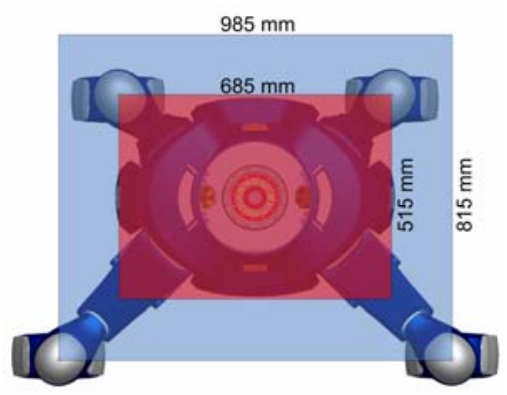

Fig. 3: Maximum and minimum footprint of the platform

With this mechanism to adjust the footprint the Rollin' Justin can do fast or heavy manipulation and high speed driving but nevertheless remains useful for indoor locomotion.

In order to obtain a stable stand with four wheels on the ground especially on uneven terrain, the wheel suspensions are individually equipped with a spring damper system. In this way, also small obstacles like doorsteps can be passed in a gentle way. The height of the platform in a neutral state of the upper body is $693 \mathrm{~mm}$. The spring can be deflected $35 \mathrm{~mm}$ around its equilibrium.

\section{A. Wheels and Steering}

The platform is driven by four independent hub motors (cf. Fig. 4). These are identical brushless-DC direct drives with a maximum torque of $30 \mathrm{Nm}$. They are driving a steel rim with thin $(5 \mathrm{~mm})$ solid rubber tires that have a diameter of $216 \mathrm{~mm}$. With this combination the platform can reach a velocity of $1.5 \mathrm{~m} / \mathrm{s}(5.4 \mathrm{~km} / \mathrm{h})$. Due to the diameter of the tires and the torque of the wheel motors the Rollin' Justin (over all weight: $199 \mathrm{~kg}$ ) can pass steps up to $40 \mathrm{~mm}$ and climb ramps up to a slope of 17 degrees. The advantages of hub motors are firstly that the space inside the wheels can be used and thus the demand for a compact design is supported, and secondly the center of gravity is lowered due to the weight of the motors that is located near the ground. This supports the demand for stability.

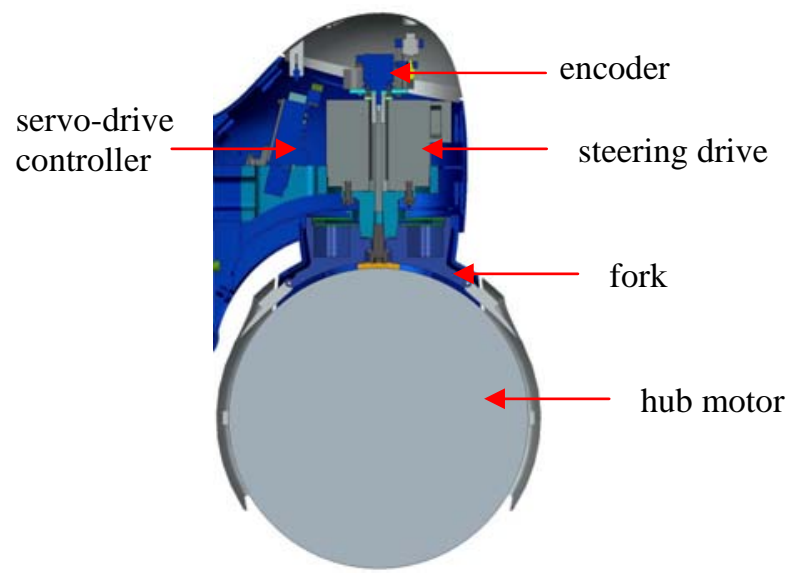

Fig. 4: Cross section of the wheel unit
A single-sided fork attaches the wheel to the steering drive. The steering drive is a type FHAC-mini motorgearbox unit from Harmonic Drive, consisting of a brushless DC motor and a 100 -fold reducing harmonic drive gearbox. The maximum steering speed is $360 \% \mathrm{~s}$ and the maximum torque is $28 \mathrm{Nm}$. The FHAC-mini is a high integrated offthe-shelf drive unit that meets the requirements on torque and velocity demanded on base of experiments with the mobile platform from the DLR project Robutler [10].

A type Whistle digital servo-drive controller from Elmo Motion Control is driving the steering motor. It is located next to the steering drive together with the filter and interface electronics of the motor controller. An additional type RE22S absolute encoder from Renishaw provides the absolute steering position at the output of the gearbox.

\section{B. Wheel Suspension}

Each of the four wheel units, consisting of hub motor, steering drive, servo-drive controller and absolute encoder, is mounted on a special suspension: A parallel mechanism with a spring damper element. Fig. 5 shows the cross section of an entire leg of the platform consisting of the wheel unit, the suspension with parallel mechanism and spring damper unit and the leg carrier with attached guide rails. The retracted position is shown up in black outlines.

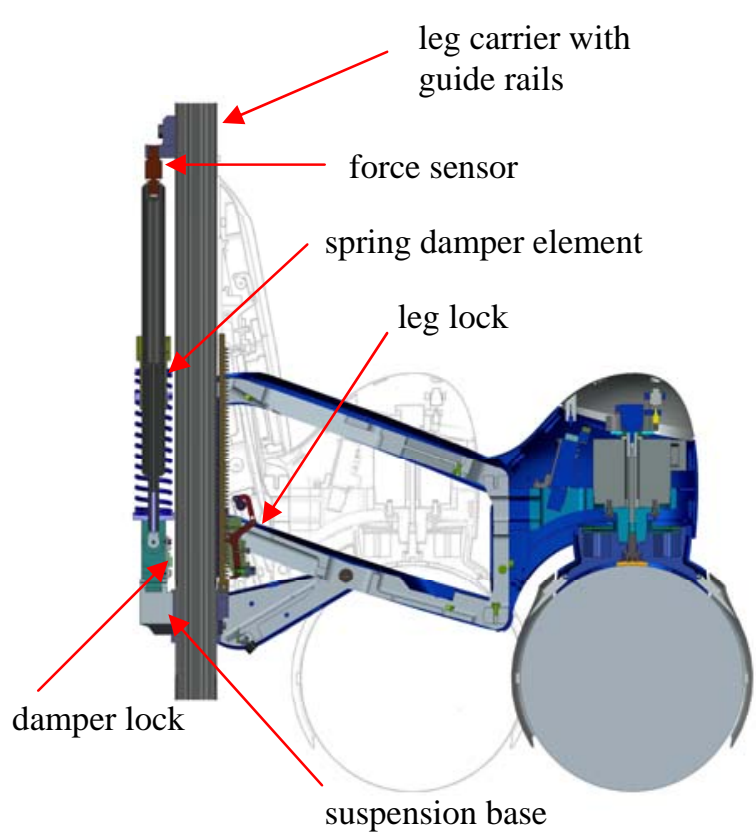

Fig. 5 Cross section of the leg with wheel,
steering motor, parallel kinematics and
spring damper element

The parallel mechanism enables the wheel to move in and out in horizontal direction without moving in vertical direction. The result is that the platform can vary its footprint while height and inclination remain unchanged. The mechanism is passive and actuated by appropriate wheel motion. To avoid unintentional extension or retraction of the wheel the parallel mechanism can be locked (leg lock). A small servo motor that is programmed for two different 
positions opens and closes the lock of a toothed rack that connects the suspension base with the slider of the parallel mechanism. Slider and base are both mounted via linear bearings on guide rails which are attached to the leg carrier.

Due to the mounting of the suspension base on the same guide rails as the slider of the parallel mechanism the entire wheel unit can move up and down the leg carrier. A spring damper element is connecting the suspension base to the leg carrier. The travel of the damper between its hardware limits is $70 \mathrm{~mm}$. They are experimentally tuned to get the best performance in passing doorsteps and stabilizing the platform. The spring has a length of $170 \mathrm{~mm}$, a spring constant of $5.47 \mathrm{~N} / \mathrm{mm}$ and a pre-load of $24 \mathrm{~mm}$. So the balance point of the four spring damper units carrying the Rollin' Justin is nearly in the middle of the damper travel and the platform can pass steps up to $35 \mathrm{~mm}$ without reaching the hardware limits. The used dampers, type K0N9KX2-070 from Bansbach, are provided with a locking function. Another small servo motor releases or locks the damper by pushing or releasing a button on top of the piston rod. With locked dampers the platform behaves like a rigid base with high stiffness for Justin. This is recommended for manipulation tasks [11]. With released dampers the platform behaves like a car. It always has a stable stand with four wheels on the ground, can pass small steps and pitches slightly with changes of the center of gravity or acceleration.

Between the damper and the fixing to the leg carrier there is a force sensor in each leg with a range of $0-$ $1000 \mathrm{~N}$. By measuring the weight on each wheel it is possible to determine the center of gravity. Together with an inertia unit in the head of Justin it can be tried to identify the stiffness parameters of the locked or unlocked platform, and a compensation as realized for the light-weight arms and torso can be implemented.

\section{Platform Body}

All four legs of the platform are attached to a frame that is the basic part of the platform. The frame together with the legs builds the chassis of the platform that is shown in Fig. 6.

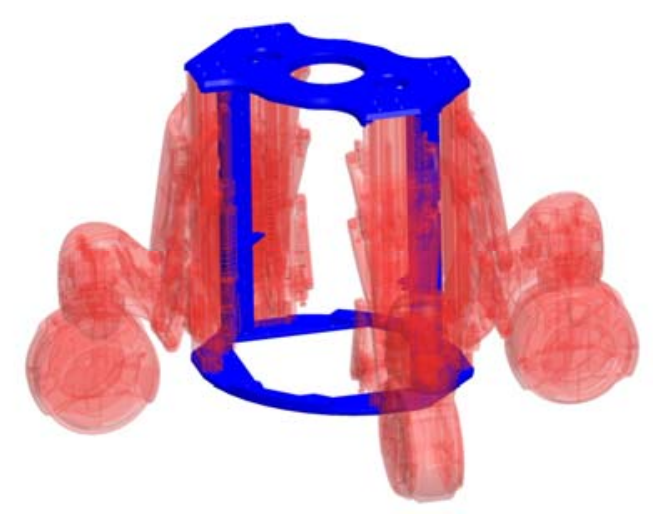

Fig. 6 Platform chassis, body frame (blue) with mounted legs

Apart from the function as leg carrier, the frame is also the carrier for every other part of the platform. The result is a very modular assembly of the whole vehicle. Every leg and nearly every electronics component inside the platform can be changed independently from other components. This simplifies maintenance work.

The inside of the chassis is filled with the battery, the power supply, several computers, the hardware infrastructure and a user interface for the main controls of the system (cf. Fig. 7). The available space for the hardware is about 50 liters.

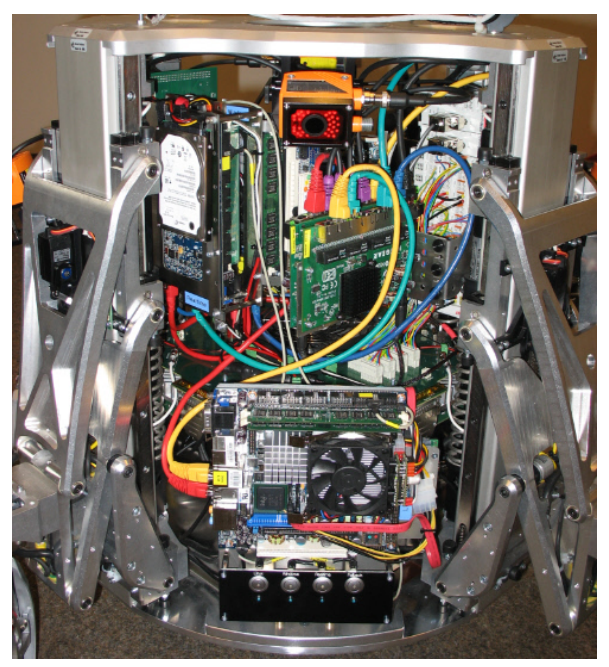

Fig. 7: The platform entirely filled with electronics

\section{ELECTRONICS AND SUPPLY}

When constructing a mobile robot one of the most difficult tasks is to integrate the entire hardware for controlling the robot and especially the power supply onboard. Many experimental mobile robots still have a connection to the environment via cable either for power supply or for realtime or high-speed communication [6] [12] [13]. But true mobility can only be reached when a robot can act completely autonomously without physical connection to a fixed place.

So the mobile platform for Justin has to carry a proper power supply, four different computers for the control of Justin and the platform, all the infrastructure like network switches for the computers, a WLAN connection bridge, a radio receiver for emergency stop and of course the electronics of the platform itself like sensors, motor drives, realtime bus coupler, cameras, etc.

\section{A. Sensors}

As the platform should be able to localize and navigate itself safely in an unstructured environment, several sensors are needed that collect the required data. Besides the navigation based on odometry data from the wheel movement (absolute encoders for the steering angles, velocity of the wheel, absolute encoders for the wheel extensions) the platform has two video cameras for visual odometry. One camera faces to the front of the platform, the other one to the left side. Based on the changes in the captured scenes per time increment, also called optical flow, 
the movement of the platform can be calculated. The result is a redundant odometry with minimized error.

Simultaneous localization and mapping (SLAM) and collision prevention can be performed using the data of photo mixer device (PMD) cameras. These cameras provide a depth value for every captured pixel in the scene. This delivers a 3D view of the environment around the platform. Four PMD cameras are observing the four main directions front, left, right and back. In this configuration the platform is able to detect walls, doors, obstacles as well as dynamical objects like human legs.
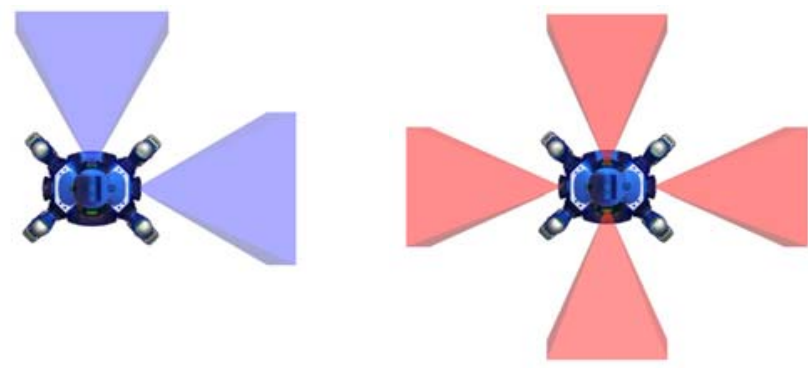

Fig. 8: Field of view of the video cameras (left) and the PMD-cameras (right)

For additional safety issues every wheel has bumper switches to the forward and backward direction of the wheel. Since the wheels are the outer parts of the platform a physical contact with an obstacle will always be at the bumper plains. The area between the legs is supervised by the PMD-cameras. If one of the bumpers gets pushed, the platform can react by stopping immediately. But this is just a safety option as in normal case the platform should detect and avoid all obstacles.

\section{B. Power Supply}

The power supply consists of a rechargeable battery as energy storage and the supply circuit to provide the different components with a proper voltage level. It is one of the most important hardware parts that has to be very reliable and failsafe.

\section{Battery}

The battery is a $48 \mathrm{~V}$ nominal voltage Lithium-Polymer accumulator that has a capacity of $40 \mathrm{Ah}$. The maximum discharge current is $200 \mathrm{~A}$ and the maximum charging current is $80 \mathrm{~A}$. The battery is made up of 13 cells in series with $3.7 \mathrm{~V}$ nominal voltage each. With an average power consumption of the entire Rollin' Justin of about 600W the battery is able to provide the system for about three hours. The recharge can be done within one hour at maximum charging current.

A balancing and safety electronic is monitoring and correcting the operating parameters (voltage, current, temperature) of each cell. Due to the very high energy density of Lithium-Polymer cells the battery, together with its monitoring electronics and a protective cover of stainless steel, takes just 13 liters of the available space inside the platform.

\section{Supply circuit}

The battery voltage fluctuates with the charge condition between $40 \mathrm{~V}$ and $54 \mathrm{~V}$. For the different robot components this main supply has to be converted into proper voltage levels. Six separated power lines are providing all the components at their individual specified voltage level (48 V, $3 \mathrm{x} 24 \mathrm{~V}, 12 \mathrm{~V}, 5 \mathrm{~V}$ ). To cope with short circuits and over current errors every power line is fused at least once. For analysis and check up purposes the current is measured separately in every supply line and shown at a status display. The supply circuit can handle a maximum (continuous) power of $3.7 \mathrm{~kW}$.

\section{Emergency Stop, Computers and Peripherals}

As the mobile platform together with Justin is meant to be autonomous, it must not have any cable connection. To be able to stop the system in case of error anyway, a wireless emergency stop concept is required.

A radio and infrared receiver on the platform controls a current loop that delivers an "OK"-signal to the relevant components (motor controllers and supplies) of the robot. As long as the receiver gets a coded message of the transmitter unit, the "OK"-signal is true. To be robust against disturbances the transmitter is sending on two different radio frequencies (433MHz and $868 \mathrm{MHz}$ ) and via infra red. It can be turned on and off by an emergency button. The timeout of the receiver is set to $200 \mathrm{~ms}$.

There are four MiniITX standard PCs with dual core processors on board of the platform for control, communication and peripheral I/O. The computers are connected to each other via Gigabit-Ethernet. The Ethernet connection is done by an 8-port gigabit switch and the uplink to the control interface outside the platform is done by a Wireless-LAN bridge. The video cameras and the PMD cameras are attached via a second 8-port gigabit switch.

The list of electronics on board the platform is completed by the peripherals like firewire hubs, additional small supply units, USB controller for the user interface, different antennas for WLAN and emergency stop receiver, analog-digital converters for the force sensors, SSIinterfaces for the absolute encoders, controller electronics for the locking servos, several fans for cooling the interior, etc.

\section{COMMUNICATION}

The second important design guideline building the platform was a compact construction and the third using offthe-shelf components. Therefore the components were chosen upon their size and suitability for the functional needs but not on the homogeneity of bus and communication protocols. This requires the communication concept to cope with many different realtime protocols and bus systems.

The hub motor wheels are communicating via CAN 2.0B at a command rate of $16 \mathrm{~ms}$. The digital servo drivers (Whistle) for the steering motors are using CANopen (4 ms). The absolute encoders for the steering angle and the leg extension deliver their values via an SSI connection and the values from the force sensors have to be read by an analog-digital-converter. The position of the servo drives for 
the locking functions in the wheel suspensions can be commanded by a digital output signal. Arms, torso and head of Justin are using the realtime bus SERCOS and the hands use HIC (Heterogeneous Interconnect, IEEE 1355). The whole upper body system has a control loop rate of $1 \mathrm{kHz}$ [9].

Similar to the Justin control concept all the sensor data should be available in hard realtime on a single PC where the robot can be used within a Simulink environment as hardware in the loop system. Normally this would require a lot of driver programming, several PCI slots and cards for the different bus systems and a lot of CPU power consumed by data acquisition, which makes the design of the control system difficult and error-prone.

For Rollin' Justin a commercially available PLC communication concept from Beckhoff is used. A single PC is dedicated as data collector platform which retrieves the data from the CAN 2.0b, the OpenCAN, the analog, the SSI and the SERCOS PLC components and transfers them in realtime to an EtherCAT bus. The data collector acts as EtherCAT Master. All the timing constraints and data conversions are configured using the standard software TwinCAT from Beckhoff. On the dedicated control PC for the platform and torso control only an Ethercat Slave PCI card is present, providing all sensor data of the entire system via mapped memory. Therefore the communication overhead on the realtime control PC is small and there is only one fairly simple driver for the EtherCat slave which has to be programmed. On the data collector PC however, the communication overhead is quite high but there an industry approved system is used. No driver has to be programmed. Only PLC components to start and stop the communication system have to be configured, which is also sometimes a challenge, but less work intensive than programming the protocol drivers itself. Fig. 9 gives an overview about the realtime communication concept of the Rollin’ Justin.

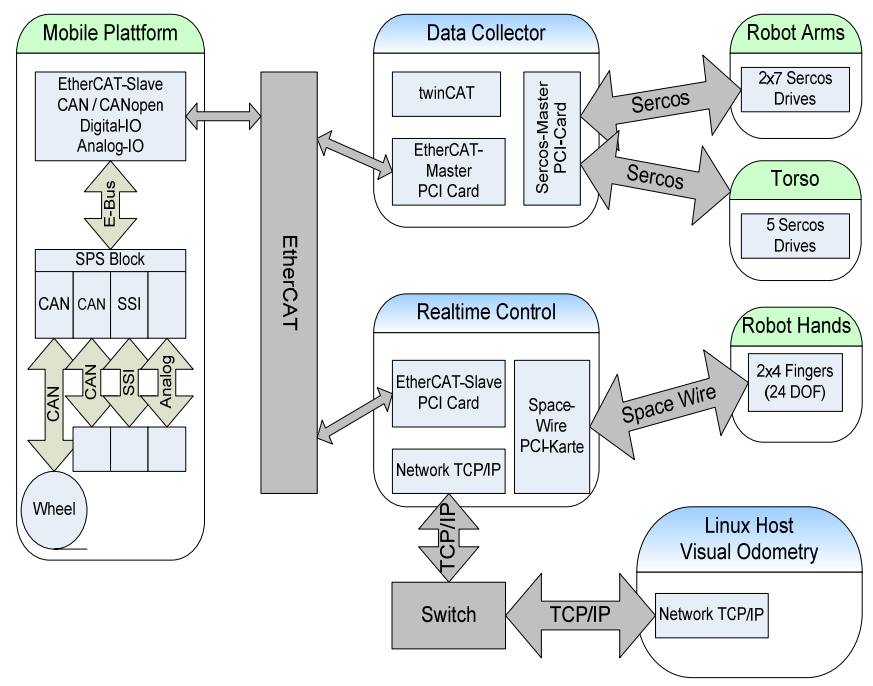

Fig. 9: Realtime communication concept of the Rollin’ Justin

The control of the upper body runs in a $1 \mathrm{kHz}$ loop while the control of the platform is slower.
Besides the realtime part there are also components that do not need to communicate in realtime. Video cameras, PMD cameras, inclination sensor and status display are connected to the standard I/O interfaces (Ethernet, USB, IEEE 1394) of the Linux computer. The Linux PC is also the gateway to the control hosts outside the platform attached via WLAN interface.

\section{Platform Control}

Mobile platforms equipped with several steering wheels are known to be omnidirectional, i.e., able to independently translate and rotate on the plane [14]. As explained in Sec. III, Justin's mobile platform also possesses the ability to vary its footprint over time by extending or retracting the wheels, also during motion of the platform. Therefore, we aimed at obtaining a control algorithm able to track an arbitrary (linear/angular) planar trajectory while, at the same time, imposing an independent and decoupled extending or retracting motion to each leg. This is achieved in two steps:

Firstly, a suitable kinematic model representing the special leg actuation mechanism is derived. Then, upon this model, a tracking controller is designed able to realize the proposed task by properly actuating the rolling and steering velocities of the wheels. A nice property of this feedback is that it dynamically linearizes the model equations, so that decoupled and exponential convergence for the tracking error can be easily obtained. A full description of both modeling and control issues, together with an illustration of experimental results, can be found in [15].

\section{CONCLUSION}

This paper presents the design and realization of Rollin' Justin's mobile platform. The mechanical assembly with parallel mechanism, spring-damper unit, hub motor, steering drive and platform body is described as well as the structure of the electronics with the different sensors, the supply circuit, the computers and the battery. A novel way of integrating mechatronical components using several different realtime busses and protocols is shortly presented.

With Rollin' Justin a valuable robotic system is available for research on sophisticated mobile two-handed manipulation. This enables the development of strategies for complex manipulation or fetch and delivery tasks. These are typical demands for e.g. future service robotic applications done by humanoid robots.

\section{REFERENCES}

[1] A. Wilhelm, "Design of a Mobile Robotic Platform with Variable Footprint,” Master's Thesis, 2007, University of Waterloo, Canada.

[2] Brian H. Wilcox, Todd Litwin, Jeff Biesiadecki, Jaret Matthews, Matt Heverly, Jack Morrison, Julie Townsend, Norman Ahmed, Al Sirota, Brian Cooper, "ATHLETE: A Cargo Handling and Manipulation Robot for the Moon," Journal of Field Robotics 24(5), DOI: 10.1002/rob.20193, 2007, 421-434.

[3] Y. Takita, N. Shimoi, H. Date, "Development of a wheeled mobile robot 'octal wheel' realized climbing up and down stairs”, Proc. IEEE Conf. on Intelligent Robots and Systems, 2004

[4] Bischoff, R. "HERMES - A Humanoid Mobile Manipulator for Service Tasks,” Proc. Int. Conf. Field and Service Robots, Canberra, Australia, 1997. 
[5] T. Asfour, K. Regenstein, P. Azad, J. Schröder, A. Bierbaum, N. Vahrenkamp, R. Dillman, "ARMAR-III: An Integrated Humanoid Plattfrom for Sensory-Motor Control”, Proceeding, Humanoids 2006, International Conference on Humanoid Robots, December 2006, Genoa, Italy

[6] “Twendy-One," Sugano Laboratory, WASEDA University, online: http://www.twendy-one.com, (Sept. 2008).

[7] R. Holmberg, O. Kathib, "Development and Control of a Holonomic Mobile Robot for Mobile Manipulation Tasks”, Int. Journal of Robotics Research, 19(11), 2000

[8] Goza, S., Ambrose, R., Diftler, M., Spain, I., 2004, "Telepresence Control of the NASA/DARPA Robonaut on a Mobility Platform," Proc. SIGCHI Conf. on Human Factors in Computing Systems, pp. 623-629.

[9] Ch. Ott, O. Eiberger, W. Friedl, B. Bäuml, U. Hillenbrand, Ch. Borst, A. Albu-Schäffer, B. Brunner, H. Hirschmüller, S. Kielhöfer, R. Konietschke, M. Suppa, T. Wimböck, F. Zacharias, and G. Hirzinger, „A Humanoid Two-Arm System for Dexterous Manipulation,“ in IEEE Int. Conf. on Robotics and Automation, 2007.

[10] Ch. Ott, Ch. Borst, U. Hillenbrand, B. Brunner, B. Bäuml, G. Hirzinger, "The Robutler: Towards Service Robots for the Human Environment,” in: CD ICRA 2005, S. 1399 - 1405, IEEE Int. Conf. on Robotics and Automation, Barcelona, Spain, 2005

[11] Ott, Christian; Albu-Schaeffer, Alin; Hirzinger, Gerhard, "A Cartesian Compliance Controller for a Manipulator Mounted on a Flexible Structure,” in: Proceedings, IEEE/IROS International Conference on Intelligent Robots and Systems, S. 4502 - 4508, RSJ, Beijing, 2006.

[12] M. Görner, T. Wimböck, A. Baumann, M. Fuchs, T. Bahls, M. Greben-stein, Ch. Borst, J. Butterfass and G. Hirzinger, „The DLR Crawler: A Testbed for Compliant Hexapod Walking Based on the DLR Hand II Fingers," in IEEE/RSJ Int. Conf. on Intelligent Robots and Systems, 2008.

[13] Andreas Birk and Cosmin Condea, "Mobile Robot Communication without the Drawbacks of Wireless Networking," RoboCup 2005: Robot WorldCup IX, I. Noda, A. Jacoff, A. Bredenfeld, Y. Takahashi (Eds.) Lecture Notes in Artificial Intelligence (LNAI) 4020, pp. 585 592, Springer, 2006.

[14] B. Thuillot, B. d'Andréa Novel, and A. Micaelli, "Modeling and feedback control of mobile robots equipped with several steering wheels," IEEE Trans. on Robotics and Automation, vol. 12, no. 3, pp. 375-290, 1996.

[15] P. Robuffo Giordano, M. Fuchs, A. Albu-Schaeffer, and G. Hirzinger, "On the Kinematic Modeling and Control of a Mobile Platform Equipped With Several Steering Wheels and Movable Legs," submitted to the 2009 IEEE Int. Conf. on Robotics and Automation, 2009. 